TRANSACTIONS OF THE

AMERICAN MATHEMATICAL SOCIETY

Volume 275, Number 2, February 1983

\title{
WEIGHTED NORM INEQUALITIES FOR HOMOGENEOUS FAMILIES OF OPERATORS
}

BY

JOSE L. RUBIO de FRANCIA

\begin{abstract}
If a family of operators in $R^{n}$ is invariant under rotations and dilations and satisfy a certain inequality in $L^{p}\left(l^{r}\right)$, then it is uniformly bounded in the weighted space $L^{r}\left(|x|^{n(r / p-1)} d x\right)$. This is the main consequence of a more general result for operators in homogeneous spaces. Applications are given to certain maximal operators, the Fourier transform and Bochner-Riesz multipliers.
\end{abstract}

1. Introduction. In [17], it was shown how the theory of factorization of operators through $L^{p}$ spaces, together with certain known vector valued inequalities, could be used to solve the problem of finding those $v(x) \geqslant 0$ (resp. $w(x) \geqslant 0)$ such that

$$
\int|T f(x)|^{p} v(x) d x \leqslant\left.\int|f(x)|\right|^{p} w(x) d x
$$

for some nontrivial $w(x)$ (resp. $v(x)$ ), where $T$ is a certain operator in $L^{p}\left(R^{n}\right)$. Here we go a step further to obtain concrete weights for which the inequality holds, namely

$$
\int|T f(x)|^{p}|x|^{a} d x \leqslant \int|f(x)|^{p}|x|^{a} d x
$$

The general result of this kind is Theorem 3, and it is applied to obtain weighted norm inequalities for some operators (or families of operators) in $R^{n}$ invariant under dilations and rotations, with weights of the form $|x|^{a}$. A more general result (Theorem 1) is actually proved, which may be also applied to translation invariant families of operators in an amenable group. The operators to which the method applies must be linearizable in the following sense.

Definition. An operator $T$ from a Banach space $B$ to the space $L^{0}(\mu)$ of all $\mu$-measurable functions is linearizable if, for every $f \in B$ there exists a linear operator $U=U_{f}: B \rightarrow L^{0}(\mu)$ such that

$$
|U f(x)|=|T f(x)| ;|U g(x)| \leqslant|T g(x)| \quad(\forall g \in B) .
$$

This defines all the interesting operators in Fourier analysis: linear operators, $g$-functions, maximal operators, etc.

Received by the editors October 6, 1981 and, in revised form, February 23, 1982.

1980 Mathematics Subject Classification. Primary 42B25, 42B15, 43A85.

Key words and phrases. Weighted norm inequalities, rotation and dilation invariant operators, vector valued inequalities, amenable groups. 
2. Main results. Let $G$ denote a locally compact amenable group with left Haar measure $m$, and consider a compact subgroup $K$ of $G$. We form the locally compact (homogeneous) space $X=G / K$ consisting of all right cosets $K g(g \in G)$. Then $G$ acts on $X$ in a natural way: If $x=K h \in X$ and $g \in G$, then $x \cdot g=K h g \in X$. A regular measure $d x$ is defined on $X$ as follows. We fix a continuous homomorphism $\alpha: G \rightarrow R_{+}=(0, \infty)$ and take the image of the measure $\alpha(g) d m(g)$ under the natural projection $\pi: G \rightarrow G / K$, i.e.

$$
\int_{X} f(x) d x=\int_{G} f(K g) \alpha(g) d m(g)
$$

for every continuous $f$ with compact support. Since $K$ is compact, $\alpha$ is constant on each coset $\pi^{-1}(x)$, and its constant value will be denoted by $\alpha(x)$. In this way, we have also defined $\alpha$ on $X$, and (1) can be written

$$
\int_{X} f(x) \alpha(x)^{-1} d x=\int_{G} f(K g) d m(g) .
$$

For functions $f$ and operators $T$ on $X$, we denote

$$
f^{h}(x)=f(x \cdot h), \quad T^{h} f=\left(T f^{h^{-1}}\right)^{h} \quad(h \in G) .
$$

THEOREM 1. Let $\mathscr{F}$ be a family of linearizable operators in $L^{p}(X, d x), 1<p<\infty$, which is $G$-invariant, i.e. $T \in \mathcal{F}$ implies $T^{h} \in \mathscr{F}$ for all $h \in G$. Suppose that, for some $r, 1 \leqslant r<\infty$, the following vector valued inequality holds

$$
\left\|\left(\sum_{j}\left|T_{j} f_{j}\right|^{r}\right)^{1 / r}\right\|_{L^{p}(d x)} \leqslant C\left\|\left(\sum_{j}\left|f_{j}\right|^{r}\right)^{1 / r}\right\|_{L^{p}(d x)} \quad\left(T_{j} \in \mathcal{F}\right) .
$$

Then, the family $\mathcal{F}$ is uniformly bounded in the weighted space $L^{r}\left(X, \alpha(x)^{r / p-1} d x\right)$, i.e.

$$
\int|T f(x)|^{r} \alpha(x)^{r / p-1} d x \leqslant C^{r} \int|f(x)|^{r} \alpha(x)^{r / p-1} d x \quad(T \in \mathscr{F}) .
$$

Proof. Consider first the case $1 \leqslant r<p<\infty$, and denote $s=p / r$. The main point in the proof is that (3) implies the following: For every $u \in L^{s^{\prime}}(X, d x)$, there exists $U \in L^{s^{\prime}}(X, d x)$ such that $\|U\|_{s^{\prime}} \leqslant\|u\|_{s^{\prime}}$ and

$$
\int_{X}|T f(x)|^{r} u(x) d x \leqslant C^{r} \int_{X}|f(x)|^{r} U(x) d x \quad(T \in \mathscr{F}) .
$$

This is a particular case of [17, Theorem A], and it can be obtained from Maurey's results on factorization of operators through $L^{r}$ (see [15]). Now, for every $h \in G$, we can replace in (5) $f(x)$ and $T f(x)$ by $f\left(x \cdot h^{-1}\right)$ and $T f\left(x \cdot h^{-1}\right)$ respectively (due to the invariance of $\mathscr{F}$ ). Since

$$
\begin{aligned}
\int_{X} \psi(x \cdot h) d x & =\int_{G} \psi(K g h) \alpha(g h) \alpha(h)^{-1} d m(g) \\
& =\alpha(h)^{-1} \Delta(h) \int_{X} \psi(x) d x
\end{aligned}
$$

where $\Delta$ is the modular function of $G$, we obtain from (5)

$$
\int_{X}|T f(x)|^{r} u(x \cdot h) d x \leqslant C^{r} \int_{X}|f(x)|^{r} U(x \cdot h) d x \quad(T \in \mathscr{F} ; h \in G) .
$$


Let $F$ be any compact subset of $X$. Since the subgroup $K$ is compact, $\tilde{F}=\pi^{-1}(F)$ is a compact subset of $G$, and since $G$ is amenable, there exists an open set $V$ in $G$ such that $m(\tilde{F} V) \leqslant(1+\varepsilon) m(V)$ (see [8]). Then, if we take $u(x)=\alpha(x)^{-1 / s^{\prime}} \chi_{F \cdot V}(x)$ (where $F \cdot V=\{y \cdot h \mid y \in F, h \in V\} \subset X$ ) it follows that

$$
\begin{aligned}
\|U\|_{s^{\prime}} & \leqslant\|u\|_{s^{\prime}}=\left(\int_{G} \chi_{F \cdot V}(K g) d m(g)\right)^{1 / s^{\prime}} \\
& =m(\tilde{F} V)^{1 / s^{\prime}} \leqslant\{(1+\varepsilon) m(V)\}^{1 / s^{\prime}} .
\end{aligned}
$$

Now we multiply both sides of (6) by $\alpha(h)^{1 / s^{\prime}}$ and integrate with respect to $h$ over the open set $V$. In the left hand side we get for every $x \in F$

$$
\begin{aligned}
\int_{V} u(x \cdot h) \alpha(h)^{1 / s^{\prime}} d m(h) & =\alpha(x)^{-1 / s^{\prime}} \int_{V} \chi_{F \cdot V}(x \cdot h) d m(h) \\
& \geqslant m(V) \alpha(x)^{-1 / s^{\prime}} .
\end{aligned}
$$

In the right hand side, by using (1) and the left invariance of Haar measure, we get

$$
\begin{aligned}
\int_{V} U(x \cdot h) \alpha(h)^{1 / s^{\prime}} d m(h) & \leqslant m(V)^{1 / s} \alpha(x)^{-1 / s^{\prime}}\left(\int_{G} U(x \cdot h)^{s^{\prime}} \alpha(x \cdot h) d m(h)\right)^{1 / s^{\prime}} \\
& =m(V)^{1 / s} \alpha(x)^{-1 / s^{\prime}}\|U\|_{s^{\prime}} \leqslant(1+\varepsilon) m(V) \alpha(x)^{-1 / s^{\prime}} .
\end{aligned}
$$

Combining everything

$$
\int_{F}|T f(x)|^{r} \alpha(x)^{-1 / s^{\prime}} d x \leqslant C^{r}(1+\varepsilon) \int_{X}|f(x)|^{r} \alpha(x)^{-1 / s^{\prime}} d x \quad(T \in \mathscr{F}) .
$$

Since the compact set $F$ and $\varepsilon>0$ are arbitrary, the theorem is proved in this case.

Now we consider the case $1<p<r<\infty$. Let $\mathcal{Q}$ be the family of all linear operators in $L^{p}(X, d x)$ which are dominated by some operator in $\mathscr{F}$, i.e. $U \in \mathcal{Q}$ iff there exists $T \in \mathcal{F}$ such that

$$
|U f(x)| \leqslant|T f(x)| \text { for all } f \in L^{p}(d x) .
$$

It is clear that the operators in $\mathscr{Q}$ satisfy the inequality (3). By duality, the adjoint operators $\left\{U^{*} \mid U \in \mathcal{Q}\right\}$ satisfy the vector valued inequality in $L^{p^{\prime}}\left(l^{r^{\prime}}\right)$, and by the case already proved

$$
\int\left|U^{*} f(x)\right|^{r^{\prime}} \alpha(x)^{r^{\prime} / p^{\prime}-1} d x \leqslant C^{r^{\prime}} \int|f(x)|^{r^{\prime}} \alpha(x)^{r^{\prime} / p^{\prime}-1} d x \quad(U \in \mathcal{Q}) .
$$

But the norm of $U^{*}$ as an operator in $L^{r^{\prime}}(w(x) d x)$ is equal to the norm of $U$ as an operator in $L^{r}\left(w(x)^{-r / r^{\prime}} d x\right)$. Therefore

$$
\int|U f(x)|^{r} \alpha(x)^{r / p-1} d x \leqslant C^{r} \int|f(x)|^{r} \alpha(x)^{r / p-1} d x \quad(U \in \mathcal{Q})
$$

and by definition of linearizable operator, the same is verified by the family $\mathscr{F}$.

As a first example of the situation described above, we can consider the group of rotations in $R^{n}, G=S O(n)$, and the compact subgroup $K=\left\{g \in G \mid x_{0} \cdot g=x_{0}\right\} \cong$ $S O(n-1)$, where $x_{o}=(1,0, \ldots, 0)$. Then $G / K$ is naturally identified with the unit sphere $\Sigma_{n-1}=\left\{x \in R^{n}|| x \mid=1\right\}$ (to each $x \in \Sigma_{n-1}$ corresponds the coset $\{g \in G \mid$ $\left.x_{0} \cdot g=x\right\}$ ). Since $G$ is compact, the only homomorphism which we can choose is 
$\alpha(g)=1$, and $d x$ is normalized Lebesgue measure in $\Sigma_{n-1}$. Then, Theorem 1 gives

THEOREM 2. Let $T$ be a linearizable operator in $L^{p}\left(\Sigma_{n-1}\right), 1<p<\infty$, and let $r \geqslant 1$ be such that

$$
\int_{|x|=1}\left(\sum_{j}\left|T f_{j}\left(\rho_{j} x\right)\right|^{r}\right)^{p / r} d \sigma(x) \leqslant C^{p} \int_{|x|=1}\left(\sum_{j}\left|f_{j}\left(\rho_{j} x\right)\right|^{r}\right)^{p / r} d \sigma(x)
$$

for all $f_{j} \in L^{p}\left(\Sigma_{n-1}\right)$ and $\rho_{j} \in S O(n)$. Then $T$ is a bounded operator in $L^{r}\left(\Sigma_{n-1}\right)$ with norm $\leqslant C$.

In the next example, $G$ will be the group of all linear transformations in $R^{n}$ which are positive multiples of a rotation, i.e.

$$
G=\left\{t \rho \mid t \in R_{+}, \rho \in S O(n)\right\} \cong R_{+} \times S O(n) .
$$

If $K$ is defined as above, $G / K$ can be identified with $R^{n}-\{0\}$. If we fix as Haar measure in $G$ the product of $d t / t$ (in $R_{+}$) and normalized Haar measure in $S O(n)$, and consider the homomorphism: $\alpha(g)=\operatorname{det}(g)=t^{n}$ (if $g=t \rho$ ), it follows very easily that $d x$ is $c_{n}$ times Lebesgue measure in $R^{n}-\{0\}$ (with $c_{n}=\Gamma(n / 2) / 2 \pi^{n / 2}$ ). On the other hand, the definition of $\alpha$ in $X$ gives in this case: $\alpha(x)=|x|^{n}$. Therefore, Theorem 1 yields

THEOREM 3. Let $\mathscr{F}$ be a family of linearizable operators in $L^{p}\left(R^{n}\right), 1<p<\infty$, which is invariant under dilations and rotations. If

$$
\left\|\left(\sum_{j}\left|T_{j} f_{j}\right|^{r}\right)^{1 / r}\right\|_{p} \leqslant C\left\|\left(\sum_{j}\left|f_{j}\right|^{r}\right)^{1 / r}\right\|_{p} \quad\left(T_{j} \in \mathcal{F}\right)
$$

holds for some $r \in[1, \infty)$, then

$$
\int_{R^{n}}|T f(x)|^{r}|x|^{n(r / p-1)} d x \leqslant C^{r} \int_{R^{n}}|f(x)|^{r}|x|^{n(r / p-1)} d x \quad(T \in \mathcal{F}) .
$$

Finally, if we apply Theorem 1 to an arbitrary $G$, and take the subgroup $K$ and the homomorphism $\alpha$ to be trivial (i.e., $K=\{e\}, \alpha(g) \equiv 1$ ), we get

THEOREM 4. Let $G$ be a locally compact amenable group, and let $T$ be a linearizable operator in $L^{p}(G), 1<p<\infty$, such that, for all $f_{j} \in L^{p}(G)$ and $h_{j} \in G$

$$
\int_{G}\left(\sum_{j}\left|T f_{j}\left(x h_{j}\right)\right|^{r}\right)^{p / r} d m(x) \leqslant C^{p} \int_{G}\left(\sum_{j}\left|f_{j}\left(x h_{j}\right)\right|^{r}\right)^{p / r} d m(x)
$$

where $r \geqslant 1$. Then $T$ is a bounded operator in $L^{r}(G)$ with norm $\leqslant C$.

When $T$ is invariant under right translations, (10) simply means that the $l^{r}$-valued extension of $T$ is bounded, i.e.

$$
\left\|\left(\sum_{j}\left|T f_{j}\right|^{r}\right)^{1 / r}\right\|_{p} \leqslant C\left\|\left(\sum_{j}\left|f_{j}\right|^{r}\right)^{1 / r}\right\|_{p}
$$

REMARK. Theorem 3 is by no means the most general result of this sort which can be obtained from Theorem 1. For instance, operators from $L^{p}$ to $L^{q}, p \neq q$, with an 
adequate homogeneity may be considered (e.g. fractional integrals). This is illustrated in the case of the Fourier transform in (3.b) below. Also, $R^{n}$ can be replaced by a nilpotent Lie group with a group of dilations acting on it (as the Heisenberg group, for instance, or $R^{n}$ itself with nonisotropic dilations).

3. Applications. Here we shall use first theorem 3 to obtain some old and new weighted norm inequalities for operators arising in Fourier analysis, with weights of the form $|x|^{a}$. Then, some results concerning translation invariant operators are obtained by using Theorem 4 .

(3.a) Maximal operators. Let $\mu$ be a positive finite Borel measure in $R^{n}$, and for each $t>0$ and $\rho \in S O(n)$, denote by $\mu_{t, \rho}$ the measure $\mu$ rotated by $\rho$ and dilated by $t$, i.e.

$$
\int f(x) d \mu_{t, \rho}(x)=\int f(t \rho x) d \mu(x) .
$$

For functions $h \in L_{+}^{1}\left(R^{n}\right)$, this definition means: $h_{t, \rho}(x)=t^{-n} h\left(\rho^{*} x / t\right)$. Associated to $\mu$ is the maximal function

$$
M_{\mu} f(x)=\sup _{t, \rho}\left|f * \mu_{t, \rho}(x)\right|
$$

which is defined a priori on (say) Schwartz functions in $R^{n}$.

Corollary 1. If $M_{\mu}$ is bounded in some $L^{p}\left(R^{n}\right), 1<p<\infty$, with norm $C$, then

$$
\int_{R^{n}}\left|M_{\mu} f(x)\right|^{r}|x|^{a n} d x \leqslant C^{r} \int_{R^{n}}|f(x)|^{r}|x|^{a n} d x
$$

for $p \leqslant r<\infty$ and $0 \leqslant a \leqslant r / p-1$.

PROOF. The vector valued inequality

$$
\left\|\left(\sum_{j}\left|M_{\mu} f_{j}\right|^{r}\right)^{1 / r}\right\|_{p} \leqslant C\left\|\left(\sum_{j}\left|f_{j}\right|^{r}\right)^{1 / r}\right\|_{p}
$$

holds for $p \leqslant r<\infty$, since we can interpolate between the obvious result for $r=p$ and the inequality for $r=\infty$

$$
\left\|\left(\sup _{j}\left|M_{\mu} f_{j}\right|\right)\right\|_{p} \leqslant\left\|M_{\mu}\left(\sup _{j}\left|f_{j}\right|\right)\right\|_{p} \leqslant C\left\|\left(\sup _{j}\left|f_{j}\right|\right)\right\|_{p} .
$$

If we apply Theorem 3 to (12) the result is proved.

If we take as $\mu$ the rotation invariant probability measure concentrated in the unit sphere of $R^{n}$, we obtain Stein's maximal spherical means

$$
\mathfrak{R} f(x)=\sup _{t \geqslant 0}\left|\int_{|y|=1} f(x-t y) d \sigma(y)\right|
$$

which is bounded on $L^{p}\left(R^{n}\right)$ when $n \geqslant 3$ and $p>n /(n-1)$ (see [20]).

COROLlaRY 2. Let $f \in L^{r}\left(R^{n},|x|^{a} d x\right)$ where $n \geqslant 3$ and $0 \leqslant a<r(n-1)-n$. Then

$$
\lim _{t \rightarrow 0} \int_{|y|=1} f(x-t y) d \sigma(y)=f(x) \text { a.e. }
$$


and

$$
\int|\mathfrak{T} f(x)|^{r}|x|^{a} d x \leqslant C_{r, a} \int|f(x)|^{r}|x|^{a} d x .
$$

The example in [20, p. 1283] shows that inequality (13) is sharp for positive $a$. The natural conjecture is that (13) holds when $-n<a<r(n-1)-n$, and this would be true if the following best possible (see Theorem 4 ) vector valued inequality were verified:

$$
\left\|\left(\sum_{j}\left|\Re f_{j}\right|^{r}\right)^{1 / r}\right\|_{p} \leqslant C\left\|\left(\sum_{j}\left|f_{j}\right|^{r}\right)^{1 / r}\right\|_{p} \quad\left(\frac{n}{n-1}<r, p<\infty\right) .
$$

On the other hand, interpolation with the known inequalities for the HardyLittlewood maximal function proves that $\Re$ is bounded in $L^{r}\left(R^{n},|x|^{a} d x\right)$ if $n \geqslant 3$, $r-n<a<r(n-1)-n$ and $n /(n-1)<r<n$.

Similar statements can be made for the spherical means $\Re^{\alpha} f$ (see [20]) with $\alpha>0$, by taking $d \mu(x)=\Gamma(\alpha)^{-1}\left(1-|x|^{2}\right)_{+}^{\alpha-1} d x$. In this case there are also results in $R^{2}$.

In the next application we take $\mu$ uniformly distributed in the unit ball of $R^{n}$, so that $M_{\mu}$ is the Hardy-Littlewood operator in $R^{n}$

$$
M^{n} f(x)=\sup _{t>0}\left|\int_{|y| \leqslant 1} f(x-t y) d y\right| .
$$

It has been recently proved by E. M. Stein [19] that

$$
\left\|M^{n} f\right\|_{L^{p}\left(R^{n}\right)} \leqslant C_{p}\|f\|_{L^{p}\left(R^{n}\right)} \quad(1<p<\infty, n \in N)
$$

with $C_{p}$ independent of $n$. Therefore, the following is also true.

Corollary 3. If $1<r<\infty, n \in N$ and $0 \leqslant a<r-1$

$$
\int_{R^{n}}\left|M^{n} f(x)\right|^{r}|x|^{a n} d x \leqslant C_{r, a} \int_{R^{n}}|f(x)|^{r}|x|^{a n} d x
$$

where $C_{r, a}$ is independent of $n$.

If the constants in the vector valued inequalities of Fefferman and Stein [7] were independent of the dimension (for the maximal function on balls, not on cubes), Corollary 3 could be extended to $-1<a<r-1$.

Now we shall apply Corollary 2 to Córdoba's maximal operator in $R^{2}$ :

$$
M_{N} f(x)=\sup _{x \in R \in \Re_{N}} \frac{1}{|R|} \int_{R}|f|
$$

where $R_{N}=$ all rectangles in $R^{2}$ of eccentricity $N$ in arbitrary directions $\}$. It is known $[3,21]$ that the norm of $M_{N}$ in $L^{2}\left(R^{2}\right)$ is bounded by $C(\log 2 N)$. Since we can use Corollary 2 with $\mu$ uniformly distributed in the rectangle $R_{0}=[-1,1] \times[-N, N]$, we have

Corollary 4. For every $N \geqslant 1$ and $2 \leqslant r<\infty$

$$
\left(\int_{R^{2}}\left|M_{N} f(x)\right|^{r}|x|^{r-2} d x\right)^{1 / r} \leqslant C(\log 2 N)\left(\int_{R^{2}}|f(x)|^{r}|x|^{r-2} d x\right)^{1 / r}
$$

where $C$ is an absolute constant. 
There are some known results for certain $n$-dimensional analogues of the operator $M_{N}$ (see [3]) to which Corollary 2 can also be applied.

Finally, we mention that Corollary 4 holds with $M_{N}$ replaced by

$$
M_{\psi} f(x)=\sup _{\varepsilon>0}\left|\int_{R^{2}} f(x-\varepsilon y) \psi(y) d y\right|
$$

where $\psi \in L^{1}\left(R^{2}\right)$ is radial and

$$
B_{p}=\left(\int_{R^{2}}|x|^{2 p-2}|\psi(x)|^{p} d x\right)^{1 / p}<\infty
$$

for some $p, 1<p \leqslant 2$ (see [1]). In this case, the norm $C(\log N)$ in (15) is replaced by $C_{p}\left(\|\psi\|_{1}+B_{p}\right)$.

(3.b) The Fourier transform. Let $\hat{f}(y)$ denote the Fourier transform of a function $f(x)$ in $R^{n}$. Then we have Pitt's inequalities (see [18]).

COROLlaRy 5. If $1<r<\infty$ and $\max (0,2-r) \leqslant a<1$, then

$$
\int_{R^{n}}|\hat{f}(y)|^{r}|y|^{-a n} d y \leqslant C_{r, a} \int_{R^{n}}|f(x)|^{r}|x|^{n(a+r-2)} d x .
$$

Proof. Let $x^{*}$ denote the inverse of a point $x \in R^{n}-\{0\}$ with respect to the unit sphere, i.e. $x^{*}=|x|^{-2} x$. Then $T f(x)=f\left(x^{*}\right)|x|^{-n}$ is a unitary operator in $L^{2}\left(R^{n}\right)$, and it is of weak type $(1,1)$. Therefore, it is bounded in $L^{p}, 1<p \leqslant 2$, and has a bounded extension to $L^{p}\left(l^{r}\right), 1<p \leqslant r \leqslant 2$ (see [14]). Since $T$ is rotation and dilation invariant, we apply Theorem 3 to obtain that $T$ is bounded in $L^{r}\left(|x|^{b n}\right)$ when $1<r \leqslant 2,0 \leqslant b<r-1$, and this is equivalent to (16) by changing variables. The case $2 \leqslant r<\infty$ follows by duality or by using Theorem 3 for the adjoint of $T$, $T^{*} f(y)=\left(f\left(x^{*}\right)|x|^{-n}\right) \hat{(}(y)$.

(3.c) Spherical summation. Let us consider now the Bochner-Riesz operators in $R^{2}$

$$
\left(S_{R}^{\alpha} f\right)^{\wedge}(\xi)=\hat{f}(\xi)\left(1-|\xi|^{2} / R^{2}\right)_{+}^{\alpha}
$$

which, according to the theorem of Carleson and Sjölin, are uniformly bounded in $L^{p}\left(R^{2}\right)$ for

$$
\frac{4}{3+2 \alpha}=p_{\alpha}<p<p_{\alpha}^{\prime}=\frac{4}{1-2 \alpha} \quad\left(0<\alpha \leqslant \frac{1}{2}\right)
$$

We shall give a new proof of the following weighted norm inequalities due to $\mathrm{I}$. Hirschman [10].

COROLlaRy 6. Given $\alpha$ and $r$ with $|1 / r-1 / 2|<\alpha \leqslant 1 / 2$, the inequality

$$
\int_{R^{2}}\left|S_{R}^{\alpha} f(x)\right|^{r}|x|^{a-2} d x \leqslant C_{r, a} \int_{R^{2}}|f(x)|^{r}|x|^{a-2} d x
$$

holds if and only if $(1 / 2-\alpha) r<a<(3 / 2+\alpha) r$.

Proof. The usual computations with Hankel transforms shows (see [9]) that, if $f \in S\left(R^{2}\right)$ is such that $\hat{f}(\xi)=1$ in the unit ball of $R^{2}$, then $S_{1}^{\alpha} f(x) \geqslant C|x|^{-3 / 2-\alpha}$. 
Therefore (17) implies $a<(3 / 2+\alpha) r$, and by duality, the necessity of $a>$ $(1 / 2-\alpha) r$ also follows. On the other hand, the vector valued inequality

$$
\left\|\left(\sum_{j}\left|S_{R_{j}}^{\alpha} f_{j}\right|^{r}\right)^{1 / r}\right\|_{p} \leqslant C_{p, r}\left\|\left(\sum_{j}\left|f_{j}\right|^{r}\right)^{1 / r}\right\|_{p} \quad\left(p_{\alpha}<p<p_{\alpha}^{\prime}\right)
$$

holds for $r=2$ by a recent result of Córdoba and López-Melero [4], and by analytic interpolation with the trivial estimates for $\left(S_{R}^{\alpha}\right)$ when $\alpha>1 / 2$, it holds when $|1 / r-1 / 2|<\alpha$. Since $2\left(r / p_{\alpha}-1\right)=(3 / 2+\alpha) r-2$ and $2\left(r / p_{\alpha}^{\prime}-1\right)=$ $(1 / 2-\alpha) r-2$, the "if" part is now a consequence of Theorem 3 .

REMARKS. Hirschman's proof is only for $r=2$, but the case $|1 / r-1 / 2|<\alpha$ follows easily from this once the theorem of Carleson and Sjölin is known. The proof given here was sketched in [17]. It is natural to expect that (18) holds with $p_{\alpha}<r, p<p_{\alpha}^{\prime}$. If this were the case, Corollary 6 would be true for the best possible range $p_{\alpha}<r<p_{\alpha}^{\prime}$.

Another known result which can be obtained from Theorem 3 is the boundedness in $L^{r}\left(R^{n},|x|^{a} d x\right),-n<a<n(r-1)$, of every singular integral operator with kernel $K(x)=\Omega\left(x^{\prime}\right)|x|^{-n}$, where $\Omega\left(x^{\prime}\right)$ satisfies the $L^{r}$-Dini condition (this is a particular case of [11, Theorem 4]). The vector valued inequality needed to prove this is contained in the general results of Benedek, Calderón and Panzone [2].

(3.d) Translation invariance. When $T$ is a bounded linear operator in $L^{p}$, (11) holds at least for $r=2$ (see [14]), and we obtain as a particular case of Theorem 4 the following well-known result (see [5]).

COROLlARY 7. Let $G$ be a locally compact amenable group. Every bounded linear operator $T$ in $L^{p}(G)$ which is invariant under right translations, is also bounded in $L^{2}(G)$. Moreover, if $T$ is positive, then it is also bounded in $L^{r}(G)$ for every $r \geqslant 1$.

The last assertion follows because (11) holds for every $r \geqslant 1$ if $T$ is linear, positive and bounded in $L^{p}$.

Finally, we can apply Theorem 4 to the problem of extending a bounded operator $T$ in $L^{p}(G)$ to $L_{B}^{p}(G)$, where $B$ is an arbitrary Banach space. The natural extension $T_{B}=T \otimes 1_{B}$ is defined a priori in $L^{p}(G) \otimes B$

$$
T_{B}\left(\sum_{j} f_{j} \cdot b_{j}\right)=\sum_{j}\left(T f_{j}\right) \cdot b_{j} \quad\left(f_{j} \in L^{p}(G), b_{j} \in B\right) .
$$

If $T_{B}$ is continuous from $L^{p}(G) \otimes B$ to $L_{B}^{p}(G)$, then it can be extended by continuity to $L_{B}^{p}(G)$.

COROllaRY 8. Let $G$ be a locally compact amenable group, and let $T$ be a linear operator invariant under right translations. Suppose that the exact range of $p$ 's for which $T$ is bounded in $L^{p}(G)$ is known to be $p_{0}<p<p_{1}$, where $1 \leqslant p_{0}<2<p_{1} \leqslant \infty$. If $T$ has a bounded extension $T_{B}$ to $L_{B}^{p}(G)$ for some $p$, then the Banach space $B$ must be of (Rademacher) type $p_{0}+\varepsilon$ and cotype $p_{1}-\varepsilon$ (with $\left.\varepsilon>0\right)$.

Proof. Suppose that $r=\sup \{q: B$ is of type $q\} \leqslant p_{0}$. Then $l^{r}$ is finitely representable in $B$ by the theorem of Maurey and Pisier [16], and from the fact that $T$ has a 
bounded extension to $L_{B}^{p}(G)$, it follows very easily that the same is true for $l^{r}$, i.e. (11) holds. But Theorem 4 then implies that $T$ is bounded in $L^{r}(G)$, contradicting the hypothesis. The argument for the cotype is quite similar.

4. Some counterexamples. The converse of Theorem 3 is false, i.e., for a family $\mathscr{F}$ dilation and rotation invariant, (9) does not imply (8). In fact, let $H_{e}$ denote the Hilbert transform in the direction $e$ (where $e \in R^{2},|e|=1$ ). Then $\left(H_{e}\right)_{e \in \Sigma_{1}}$ is dilation and rotation invariant, but the vector valued inequality

$$
\left\|\left(\sum_{j}\left|H_{e_{j}} f_{j}\right|^{2}\right)^{1 / 2}\right\|_{p} \leqslant C\left\|\left(\sum_{j}\left|f_{j}\right|^{2}\right)^{1 / 2}\right\|_{p}
$$

is only true in the trivial case $p=2$ (see [6]). However,

$$
\int_{R^{2}}\left|H_{e} f(x)\right|^{2}|x|^{a} d x \leqslant C \int_{R^{2}}|f(x)|^{2}|x|^{a} d x
$$

provided that $-1<a<1$ (see [10]).

For a family $\mathcal{F}$ invariant under dilations but not under rotations, Theorem 3 may be false. For instance, there exist a singular integral operator $T$ in $R^{2}$ whose kernel is homogeneus: $K(x)=\Omega\left(x^{\prime}\right)|x|^{-n}$ and such that $T$ is not bounded in $L^{2}\left(R^{2},|x|^{a} d x\right)$ when $|a|>1$ (see [12]). However, $T$ is dilation invariant and satisfies the vector valued inequality (11) for all $1<p, r<\infty$ (see [2]). As a consequence of this, if we consider the (nonseparable) Hilbert space $H=l^{2}(I)$, with $I=[0,2 \pi)$, and the $\mathcal{L}(H)$-valued kernel $\tilde{K}$ defined on $\mathbf{R}^{2} \cong \mathbf{C}$ by

$$
\tilde{K}(z) h=\left(K\left(e^{i \theta} z\right) h_{\theta}\right)_{\theta \in I}, \quad h=\left(h_{\theta}\right)_{\theta \in I} \in H,
$$

we get the following

COROLlaRY 9. The singular integral operator

$$
\tilde{T} f(x)=\text { p.v. } \int_{R^{2}} \tilde{K}(x-y) f(y) d y \quad\left(f \in L_{H}^{2}\left(R^{2}\right)\right)
$$

is bounded in $L_{H}^{2}\left(R^{2}\right)$ but it is not bounded in $L_{H}^{p}\left(R^{2}\right)$ when $|1 / p-1 / 2|>1 / 4$.

If we drop in Theorem 1 the condition of being $G$ amenable, the result may be false. Actually, the first assertion of Corollary 6 is not true for the (nonamenable) group $G=S L(2, R)$ (see [13]).

\section{REFERENCES}

1. N. E. Aguilera, Maximal operators associated to radial functions in $L^{2}\left(R^{2}\right)$, Proc. Amer. Math. Soc. 80 (1980), 283-286.

2. A. Benedek, A. P. Calderón, and R. Panzone, Convolution operators on Banach space valued functions, Proc. Nat. Acad. Sci. USA 48 (1962), 356-365.

3. A. Córdoba, A note on Bochner-Riesz operators, Duke Math. J. 46 (1979), 505-511.

4. A. Córdoba and B. López-Melero, Spherical summation: A problem of E.M. Stein, Ann. Inst. Fourier Grenoble 31 (1981), 147-152.

5. M. Cowling, Some applications of Grothendieck's theory of topological tensor products in harmonic analysis, Math. Ann. 232 (1978), 273-285.

6. C. Fefferman, The multiplier problem for the ball, Ann. of Math. (2) 94 (1972), 330-336.

7. C. Fefferman and E. M. Stein, Some maximal inequalities, Amer. J. Math. 1 (1971), 107-115. 
8. F. P. Greenleaf, Invariant means on topological groups and their applications, Reinhold, New York, 1969.

9. C. S. Herz, On the mean inversion of Fourier and Hankel transforms, Proc. Nat. Acad. Sci. U.S.A. 40 (1954), 996-999.

10. I. I. Hirschman, Multipliers transformations. II, Duke Math. J. 28 (1961), 45-56.

11. D. S. Kurtz and R. L. Wheeden, Results on weighted norm inequalities for multipliers, Trans. Amer. Math. Soc. 255 (1979), 343-362.

12. A A note on singular integrals with weights, Proc. Amer. Math. Soc. 81 (1981), 391-397.

13. N. Lohué, Adv. in Math. 38 (1980), 178-221.

14. J. Marcinkiewicz and A. Zygmund, Quelques inégalités pour les opérations linéaires, Fund. Math. 32 (1939), 115-121.

15. B. Maurey, Théorèmes de factorization pour les opérateurs linéaires a valeurs dans un espace $L^{p}$, Astérisque $n^{\circ} 11$, Soc. Math. de France (1974).

16. B. Maurey and G. Pisier, Séries de variables aléatoires vectorielles indépendantes et propriétés géométriques des espaces de Banach, Studia Math. 58 (1976), 45-90.

17. J. L. Rubio de Francia, Weighted norm inequalities and vector valued inequalities, Proc. Conf. Harmonic Analysis (Minneapolis, 1978), Lecture Notes in Math., vol. 908, Springer-Verlag, Berlin and New York, 1982, pp. 86-101.

18. E. M. Stein, Interpolation of linear operators, Trans. Amer. Math. Soc. 83 (1956), 482-492.

19.

20. E. M. Stein and S. Wainger, Problems in harmonic analysis related to curvature, Bull. Amer. Math. Soc. 84 (1978), 1239-1295.

21. J. O. Stromberg, Maximal functions associated to rectangles with uniformly distributed directions, Ann. of Math. (2) 107 (1978), 399-402.

División de Matemáticas, facultad de Ciencias, Universidad Autónoma de Madrid, Madrid, SPAIN 